\title{
Cystectomy and urinary diversion in the treatment of bladder cancer without artificial respiration
}

\author{
M. Friedrich-Freksa, E. Schulz, T. Nitzke, 0. Wenzel, G. Popken \\ Department of Urology, HELIOS Klinikum GmbH Berlin (MFF, TN, OW, GP), and Department of \\ Anesthesiology, Klinikum GmbH Berlin (ES), Schwanebecker Chaussee, Berlin, Germany
}

\section{ABSTRACT}

Purpose: To assess the feasibility and performance of radical cystectomy with urinary diversion using exclusively regional anesthesia (i.e. combined spinal thoracic epidural anesthesia, CSTEA).

Materials and Methods: In 2011 radical cystectomy with extended pelvic and iliac lymphadenectomy was performed on 14 patients using urinary diversion without applying general anesthesia. Under maintained spontaneous breathing, the patients were awake and responsive during the entire procedure. Postoperatively, pain management took three days with the remaining epidural catheter before oral analgesics were administered. Mobilization and diet restoration were carried out according to the fast-track concept. Outcome measurements included operative time, blood loss, beginning of oral nutrition, beginning of mobilization, postoperative pain levels using numerical and visual analog scales (NAS/VAS), length of hospital stay.

Results: All surgical procedures were performed without any complications. The absence of general anesthesia did not result in any relevant disadvantages. The postoperative progress was normal in all patients. Particularly, cardiopulmonary complications and enteroparesis did not occur. The provided palliative care proved sufficient (NAS max. 3-4). Discharge followed 10 to 22 days after surgery. At the time of discharge, the patients described the procedure to be relatively positive.

Conclusions: Our data show that CSTEA is an effective technique for radical cystectomy, whereby spontaneous breathing and reduced interference with the cardiopulmonary system potentially lower the perioperative risks especially for high-risk patients. We recommend practice of CSTEA for radical cystectomy to further evaluate and monitor the safety, efficacy, outcomes, and complications of CSTEA.
ARTICLE INFO

\section{Key words:}

Feasibility Studies;

Cystectomy; Urinary Bladder

Neoplasms; Anesthesia, Spinal;

Anesthesia, Epidural; Patient

Safety

Int Braz J Urol. 2012; 38: 645-51

Submitted for publication: January 16, 2012

Accepted after revision: August 30, 2012

\section{INTRODUCTION}

Radical cystectomy is the most preferred procedure in the treatment of muscle-invasive bladder cancer and involves high perioperative risks. The morbidity and mortality rates of radical cystectomy with urinary diversion are caused by patient-specific, anesthesiological and surgical factors (individual patient risk profile, selection and administration of the anesthesiological pro- cedure, extension of the procedure, spread or metastasis of the bladder cancer, type of urinary diversion, surgical expertise, number of operations). The perioperative mortality rate of the cystectomy is between $2.9 \%$ and $7.7 \%(1-4)$. The perioperative morbidity rate is between $11 \%$ and $68 \%$ (3).

On many procedures, applying regional anesthesiological methods can lower perioperative risks. Regional anesthesia can be administered on different levels of the spinal cord and in different 
areas of the spinal canal. The substances applied once or continually in these regions have varying effects, effectiveness and side effects.

Regional anesthesia is administered particularly on patients with high perioperative risks. In urology, this has been predominantly the case so far in locally restricted, endourological and low-risk procedures. Comparatively, the exclusive use of regional anesthesiological procedures during extended and high-risk operations, such as radical cystectomy, has previously been seldom described (5).

To notably minimize cardiopulmonary, patient-specific and post-operative risks, a thoracic epidural catheter is frequently used in fast-track surgery. We combined optimal analgesia with one-time spinal anesthesia for the perioperative, motoric blockade and performed radical cystectomy with lymphadenectomy with urinary diversion under CSTEA without general anesthesia.

\section{MATERIALS AND METHODS}

In 2011 radical cystectomy with extended lymphadenectomy using urinary diversion was performed on 14 patients with muscle-invasive bladder cancer.

The inclusion criterion for applying CSTEA was the presence of a muscle-invasive bladder cancer. The exclusion criterion was the non-feasibility of the catheter installation.

Individual patient features, such as age, gender, body mass index, stage of tumor, comorbidity, cardiopulmonary risk assessment as well as intraoperative and postoperative parameters, such as the type of urinary diversion, operative time, blood loss, postoperative beginning of oral nutrition, postoperative beginning of mobilization, postoperative pain levels, postoperative hospital stay are indicated on Table-1.

During bridging, patients, who were orally anticoagulated (Pt. 1, Pt. 6, Pt. 8), for instance due to chronic atrial fibrillation, received perioperatively low molecular heparin according to weight (low dose molecular weight heparin); with dose reduction immediately pre- and postoperatively (10 to 12 -hour waiting period pre- and postoperatively).
Radical cystectomy with extended pelvic and iliac lymphadenectomy using urinary diversion (ileal neobladder, ileal conduit, transureterocutaneostomy) was performed in the usual open manner by means of a median lower abdominal laparotomy. A cystoprostatectomy was performed on male patients while a simultaneous hysterectomy was performed on female patients, taking the vaginal front wall. In all cases, the lymphadenectomy was extended pelvically, iliacally, presacrally up to the point of aortic bifurcation.

The applied urinary diversion was suited to the patient's age, risk factors and tumor size. Continent and incontinent urinary diversions were constructed from the terminal ileum in the form of a pouch or neobladder or a conduit with both ureters re-implanted. Simple incontinent urinary diversions were applied as transureterocutaneostomy (TUUC).

Thermoregulation was conducted with heating mats placed over and under the patient. During the entire surgical procedure, $\mathrm{CO}_{2}$, temperature and blood pressure were continually measured as well as ECG was monitored on each patient.

To monitor breathing $\left(\mathrm{O}_{2}\right.$ and $\left.\mathrm{EtCO}_{2}\right)$, we used the Smart CapnoLine Plus $\mathrm{O}_{2}$ for our patients, who were not intubated and were spontaneously breathing. They could be orally and nasally applied and used for up to six hours. The nasal tube has an $\mathrm{O}_{2}$ supply line. The Smart CapnoLine Plus $\mathrm{O}_{2}$ can be used in pain management, emergency medicine, transport and critical care.

Premedication was administered orally with 10-20 mg chlorazepate. Perioperatively, an iso-osmolar electrolyte solution of $5-10 \mathrm{~mL} / \mathrm{kg}$ $\mathrm{BW} / \mathrm{h}$ was infused intravenously. Blood pressure was regulated with an arterenol perfusor (1 $\mathrm{mg} / 50 \mathrm{~mL}$ of $0.9 \% \mathrm{NaCl}$ ).

CSTEA was administered by combining a deep thoracic epidural catheter at level T10/11 or $\mathrm{T} 11 / 12$ and a spinal anesthesia at level L3/4 or L4/5 (Figure-1).

A deep thoracic epidural catheter was placed either with the patient sitting or lying in a lateral position in two stages: by placing a local anesthesia and puncturing the epidural space at level T10 to T12 between two spinous processes 


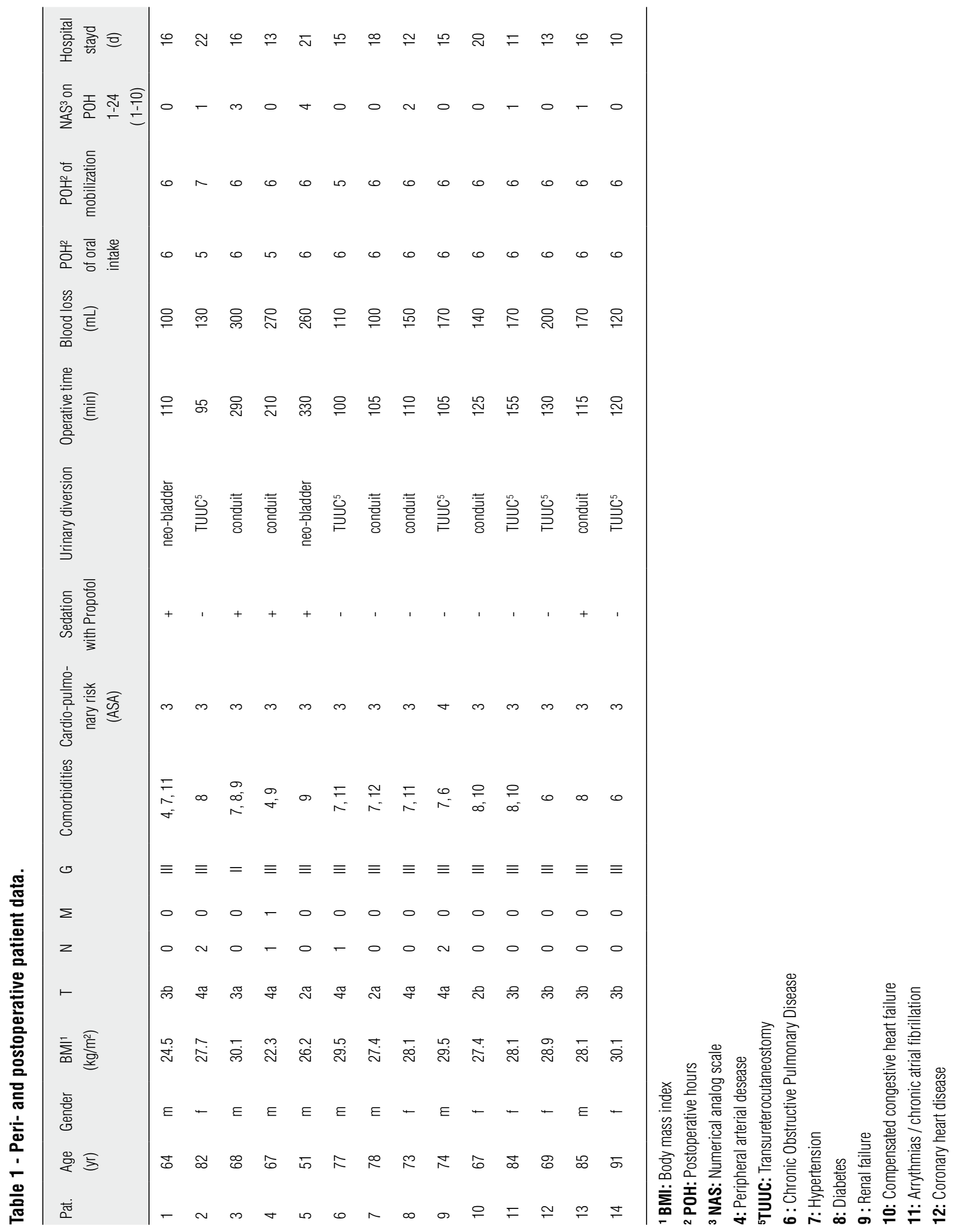


Figure 1 - Method of CSTEA.

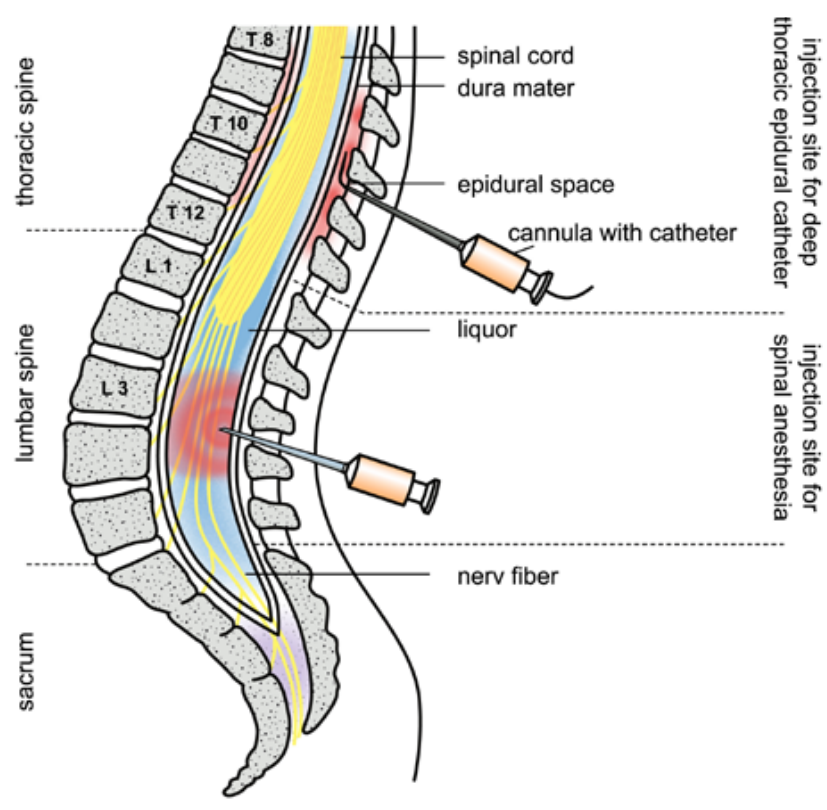

with an epidural needle (Tuohy needle, G18). The needle was inserted through the skin, the intervertebral ligaments and the ligamentum flavum in the epidural space. This was identified as the "loss-ofresistance" technique, wherein a syringe with liquid is attached to the needle. Should the needle be placed before the epidural space in the ligaments of the spinal column, fluid injection is not possible (resistance). By steadily controlling the injection resistance, the needle was carefully inserted further (usually 4-5 cm) until the resistance-free liquid could be injected (loss of resistance). This was marked by the exit of the needle point out of the ligament structure in the epidural space. After locating the epidural space, the Tuohy needle was inserted into a thin plastic catheter that could be left in the epidural space. This catheter was then tunneled under the skin. Tunneling in catheter placement supports prophylaxis against infections as well as position stability (the epidural catheter remained ca. 72 hours postoperatively, see below). Problem-free puncturing of the peridural area and placement of the catheter was tested with $0.5 \%$ bupivacaine in order to preclude the intraspinal layer. Difficult puncturing was additionally tested with adrenalin, wherein $0.5 \mu \mathrm{g} / \mathrm{kg} \mathrm{KG}$ adrenalin was injected $(10 \mu \mathrm{g} / \mathrm{mL})$.
On the actual operative procedure and beyond, the catheter enabled an effective pain management. A pump was connected to the catheter, through which a basic quantity (basal rate) of a local anesthetic was supplied continually. Postoperatively, the pumping method also aided the patient in administering additional doses, if needed, by pressing the button (patient-controlled epidural analgesia, PCEA). As long-term medication, 0.2\% (5-7 $\mathrm{mL}$ ) of ropivacaine was administered. The rapid response of epidural anesthesia followed after 5-18 minutes and its effect lasted about two hours.

Spinal anesthesia was administered on the sitting patient by puncturing with a spinal cannula (G22 with Introducer) between the lumbar vertebrae (L 3/4 or L 4/5). This was done from the rear (median) in the layers of the spinous processes. Once the cannula penetrated the ligament structure, the actual, thin puncture needle was inserted through it, thereby puncturing the subarachnoid space. After passing through the dura, the clear liquor cerebrospinalis dripped out of the needle, indicating that the needle was correctly placed. Then a local anesthetic dosed according to the patient, was administered (approximately 3 $\mathrm{mL}$ of $0.5 \%$ hyperbaric bupivacaine $(15 \mathrm{mg})$ and $5 \mu \mathrm{g}$ sufentanil). The effect was almost immediate and started with a warm sensation on the legs or buttocks. The anesthetic and analgesia settled in within minutes, while movement decreased. With the hyperbaric local anesthetic, the patient's positioning influenced the extent at which the numbed area spread. The effect lasted 3 to 4 hours.

The effectiveness of epidural and spinal anesthesia depended on the dose of the administered substance. The following nerve characteristics were disabled in this order: sensors, coldness, sympathetic, vasomotor, motor skills.

All patients respired spontaneously during the entire surgical procedure. Especially in longer operative times, some patients were administered an escalating sedation by intravenously applying propofol. For this purpose, a bolus of $10 \mathrm{mg}$ of propofol was administered followed by a propofol infusion (0.5\% propofol) of $0.5-1 \mathrm{mg} / \mathrm{kg} \mathrm{BW} / \mathrm{h}$. Postoperatively, pain therapy comprised of using the remaining epidural catheter with ropivacaine (0.1\%, 1-2 mL). 
The epidural catheter was removed after a 10 to 12 hour waiting period for heparin. We observed no epidural hematoma or other bleeding complications.

After removing the epidural catheter, pain therapy was resumed by orally administering a combination of novaminsulfon and oxycodone dosed according the patient's weight (4x500 mg / 2x10-20 mg). The pain level was documented using numerical and visual analog scales (NAS / VAS).

Postoperative mobilization and diet restoration were carried out according to the fast-track concept of early mobilization and early enteral nutrition. After surgery, patients were asked about their subjective assessment regarding the pre-, intra- and postoperative procedures.

\section{RESULTS}

Individual patient features as well as the intra- and postoperative data are summarized in Table-1.

When asked, the patients did not indicate perioperatively experiencing uneasiness, discomfort or pain.

All surgical procedures could be performed as preoperatively planned without complications and caused no anesthesiological or surgical features. Only muscle fasciculation was apparent on several patients upon severing the abdominal wall.

Vital signs of all patients were intraoperatively stable. On three patients with hypotension (side effect of the sympathetic blockade), temporarily administering a minimal dose of noradrenalin was necessary. Further side effects were not apparent.

The postoperatively continuous epidural analgesia showed a very good effect on all patients (NAS max. 3-4). After removing the epidural catheter after 72 hours, oral medication proved sufficient (NAS max. 3-4).

Postoperatively, all patients showed normal progress. Cardiopulmonary complications did not occur.

Mobilization and diet restoration were carried out according to the fast-track concept immediately postoperatively. No postoperative enteroparesis were observed. The patients could be discharged after 10 to 22 days.
When asked upon discharge, the patients described the surgical procedure pre-, intra-, postoperatively to be consistently positive under the circumstances.

\section{DISCUSSION}

To minimize perioperative risks, surgical techniques as well as preoperative anesthesiological management must be constantly improved. Better surgical techniques and anatomical understanding have continually been developed in the last years. Particularly, developments in high-frequency surgery and fast-track concepts have contributed to the quality of minimally invasive techniques. Firstly, from an anesthesiological perspective, it could be proven that applying a combination of epidural and general anesthesia is more effective than solely general anesthesia. Data from meta-analyses show that through a neuroaxial blockade, a decreased mortality rate of $30 \%$ can be reached while the morbidity rate is considerably reduced. This is achieved with a decreased incidence of deep venous thrombosis, pulmonary embolism, myocardial infarction, transfusions, pneumonias, infections, respiratory depression and liver failure $(4,6,7)$. Secondly, it is generally known that dispensing with general anesthesia leads to a clear relief of the cardiopulmonary system, from which particularly patients with increased cardiopulmonary risks benefit.

On low-risk surgical procedures in the areas of the lower abdomen and lower extremities with a sensorial blockade of up to T12 (deep spinal anesthesia) or up to T10 (medium-high anesthesia), spinal anesthesia is today considered to be an established and safe method, which as a primary indication, is applied to older and/or high-risk patients $(8,9)$. Combined spinal epidural methods (CSE methods) have the additional beneficial effect of optimal postoperative therapy over the epidurally applied local anesthetic (10). However, spinal anesthesia or CSE in the context of extensive and tumor-surgical procedures in the pelvis minor and in the lower abdomen is exceptional. Likewise, there is only few data on procedures in the area of the middle and upper abdomen, which requires a regional anesthesia of sometimes up to T4 (high spinal anesthesia). 
According to a fast-track concept, a combination of spinal singular ("single shot") and continuous thoracic epidural anesthesia is suitable for this type of surgical procedure. With the spinal application of a local anesthetic, not only rapid response and reliable analgesia are attained, but also the intraoperatively desired muscle relaxation is achieved. In contrast to continuous administration, one-time administration prevents any occurrence of longer lasting motor blockade, which impedes early mobilization (8).

The anesthesia of up to T4 is administered through the deep-thoracically placed epidural catheter. A further advantage of epidural anesthesia is the optimally postoperative pain therapy using the postoperatively remaining catheter. Different studies on intra-abdominal procedures have shown the superiority of epidural anesthesia (PCEA) to intravenous and intramuscular opioid administrations (10-12). Moreover, sparing the opioid in the first 72 hours minimizes the risk of ileus.

CSTEA without general anesthesia reduces postoperative morbidity and mortality. Moreover, it combines the advantages of spinal anesthesia with a rapid starting effect and limited effectiveness on good motor blockade with highly effective as well as optimally controllable analgesia using the postoperatively remaining epidural catheter $(6,13-16)$.

Perioperatively and postoperatively, we observed no increased complication rate regarding CSTEA and experienced no restrictions in view of pain management and early mobilization. In all cases, the administration of opioids could be dispensed with directly postoperatively.

Undesired side effects of CSTEA can include hypotension, cerebral ischemia, bradycardia, cardiac arrythmia, cardiac arrest in rare cases, respiratory insufficiency due to high spinal anesthesia, paraplegia as a result of an epidural hematoma or abscesses, arachnoidea, nausea and vomiting, motoric blockade, pruritus, post-dural puncture headache (PDPH) $(17,18)$. Intraoperatively, we observed mild hypotension on all of our patients as the only undesired side effect.

In individual cases, patients may experience unrest, which can be caused by such patient factors as longer surgery time, hypothermia, and inadequate positioning. Should this cause discom- fort or problems for the patient or the surgical team, CSTEA can be supplemented with an escalating sedation.

\section{CONCLUSIONS}

With adequate patient selection and careful application, CSTEA appears as a safe, reliable and effective option for patients undergoing radical cystectomy. Dispensing with general anesthesia resulted in no relevant perioperative disadvantages. Simultaneous, moderate sedation must be determined in individual cases.

Our data show that CSTEA is an effective technique in radical cystectomy, as particularly demonstrated by maintained spontaneous breathing and reduced interference with the cardiopulmonary system. Thus, CSTEA can be a gentle method, maybe associated with reduced perioperative risks. Larger series are necessary to prove safety, efficacy, outcomes, and complications of CSTEA, and they are under construction by our team.

\section{ABBREVIATIONS}

CSTEA: Combined spinal thoracic epidural anesthesia

CSE: combined spinal epidural

NAS: Numerical analog scale

PDPH: Post-dural puncture headache

PCEA: Patient-controlled epidural analgesia

TUUC: Transureterocutaneostomy

VAS: Visual analog scale

\section{CONFLICT OF INTEREST}

None declared.

\section{REFERENCES}

1. Finks JF, Osborne NH, Birkmeyer JD: Trends in hospital volume and operative mortality for high-risk surgery. N Engl J Med. 2011; 364: 2128-37.

2. Birkmeyer JD, Siewers AE, Finlayson EV, Stukel TA, Lucas FL, Batista I, et al.: Hospital volume and surgical mortality in the United States. N Engl J Med. 2002; 346: 1128-37. 
3. Kulkarni JN: Perioperative morbidity of radical cystectomy: A review. Indian J Urol. 2011; 27: 226-32.

4. Fairey AS, Kassouf W, Aprikian AG, Chin JL, Izawa JI, Fradet $Y$, et al.: Age $\geq 80$ years is independently associated with survival outcomes after radical cystectomy: Results from the Canadian Bladder Cancer Network Database. Urol Oncol. 2011; 1: [Epub ahead of print].

5. De Nunzio C, Cicione A, Leonardo F, Rondoni M, Franco G, Cantiani $A$, et al.: Extraperitoneal radical cystectomy and ureterocutaneostomy in octogenarians. Int Urol Nephrol. 2011; 43: 663-7.

6. Rodgers A, Walker N, Schug S, McKee A, Kehlet $H$, van Zundert $A$, et al.: Reduction of postoperative mortality and morbidity with epidural or spinal anaesthesia: results from overview of randomised trials. BMJ. 2000; 321: 1493.

7. Beattie WS, Badner NH, Choi P: Epidural analgesia reduces postoperative myocardial infarction: a meta-analysis. Anesth Analg. 2001; 93: 853-8.

8. Ballantyne JC, Carr DB, deFerranti S, Suarez T, Lau J, Chalmers TC, et al.: The comparative effects of postoperative analgesic therapies on pulmonary outcome: cumulative metaanalyses of randomized, controlled trials. Anesth Analg. 1998; 86: 598-612.

9. Denny NM, Selander DE: Continuous spinal anaesthesia. $\mathrm{Br}$ J Anaesth. 1998; 81: 590-7.

10. Goyal M, Taxak S, Kshetrapal KK, Goel MK: Continuous spinal anesthesia in a high risk elderly patient using epidural set. J Anaesthesiol Clin Pharmacol. 2011; 27: 139-41.

11. Fan SZ, Susetio L, Wang YP, Cheng YJ, Liu CC: Low dose of intrathecal hyperbaric bupivacaine combined with epidural lidocaine for cesarean section--a balance block technique. Anesth Analg. 1994; 78: 474-7.
12. Ladjevic N, Likic-Ladjevic I, Dzamic Z, Acimovic M, Dragicevic $D$, Durutovic 0: Combined general and epidural anaesthesia versus general anaesthesia for radical cystectomy. Acta Chir lugosl. 2007; 54: 89-91.

13. Werawatganon T, Charuluxanun S: Patient controlled intravenous opioid analgesia versus continuous epidural analgesia for pain after intra-abdominal surgery. Cochrane Database Syst Rev. 2005; 1: CD004088.

14. Wu CL, Cohen SR, Richman JM, Rowlingson AJ, Courpas GE, Cheung K, et al.: Efficacy of postoperative patient-controlled and continuous infusion epidural analgesia versus intravenous patient-controlled analgesia with opioids: a metaanalysis. Anesthesiology. 2005; 103: 1079-88.

15. Rawal N, Holmström B, Crowhurst JA, Van Zundert A: The combined spinal-epidural technique. Anesthesiol Clin North America. 2000; 18: 267-95.

16. Mahoney OM, Noble PC, Davidson J, Tullos HS: The effect of continuous epidural analgesia on postoperative pain, rehabilitation, and duration of hospitalization in total knee arthroplasty. Clin Orthop Relat Res. 1990; 260: 30-7.

17. Tziavrangos E, Schug AS: Regional anaesthesia and perioperative outcome. Curr Opin Anaesthesiol. 2006; 19: 521-5.

18. Parnass SM, Schmidt KJ: Adverse effects of spinal and epidural anaesthesia. Drug Saf. 1990; 5: 179-94.
Correspondence address: Dr. Male Friedrich-Freksa, Eberswalder Str. 22 Berlin, 10437, Germany Fax: +49 030 241-3155 E-mail: ffreksa@gmx.de 